\title{
Ontario funds one cycle of IVF - while supplies last
}

$\mathrm{N}$ ot all Ontarians who are eligible for a funded cycle of in vitro fertilization (IVF) will necessarily get it, and it's not clear just how the 5000 annual spots will be allocated to individual patients.

Earlier this fall, the Ontario government announced that it would start funding IVF before the end of the year, making good on a 2014 election promise. With just two weeks to go before that selfimposed deadline, however, much of the fine print has not yet been made public, including which clinics are participating, how much they will be reimbursed per procedure and whether there will be increased government oversight of the sector.

What is known is that Ontario's program will be unlike Quebec's, which was funded through the provincial health insurer (before it was scrapped in November). It will also be different from Manitoba's, which is reimbursed through a tax credit, or New Brunswick's, which offers a one-time grant of $\$ 5000$. Instead, Ontario will set aside up to $\$ 70$ million annually for specific reproductive services. Selected clinics across the province have applied and been contracted to carry out assigned quotas of the funded procedures, with numbers set by the government. Clinics will bill the ministry directly on a monthly basis.

"One of the big questions is how this is going to be allocated and whether it will be fair and consistent with human rights law," says Vanessa Gruben, a University of Ottawa law professor who specializes in reproductive health.

Deciding which patients get the funded treatments, the ministry confirms, will be left to individual clinics. Some, such as the Ottawa Fertility Centre, have already established first-comefirst-served waiting lists, with an excep- tion for cancer patients. Others reject that model. "Who is 'first'? To me that doesn't make sense," says Dr. Matt Gysler, medical director at the Reproductive Care Centre in Mississauga, Ontario. His clinic's ethics committee

transfer of any resulting embryos, mostly one at a time. Other routine IVF procedures, such as sperm injection, assisted hatching and freezing and thawing of embryos will be included in the price, but embryo storage and drugs to stimulate egg production will not.

Another procedure, intrauterine insemination (IUI), which involves delivering sperm directly into the uterus, is also included in the program. The government has contracted clinics to provide approximately 10000 funded IUI cycles.

With the launch of the funding initiative, all IUI and IVF procedures will be delisted from the Ontario Health Insurance Program (OHIP). Women with two blocked fallopian tubes, who were previously eligible for three rounds of OHIP-

will decide on a case-by-case basis, according to the clinic website.

At Toronto's Hannam Fertility Centre, most slots will be distributed by lottery, but $20 \%$ will be given to patients with particularly urgent conditions, such as impending cancer treatment or aggressive endometriosis. "It has been absolutely agonizing for us," says Hannam. "But we believe we have created a fair solution." The ministry has confirmed that patients are free to join multiple wait lists.

In 2014, some 5700 fresh IVF cycles were started in Ontario. Many now expect numbers to rise sharply. "It's the first year, so you're going to get everybody eligible - not just patients new to IVF, but all the people who did previous cycles and failed or who already have a child," speculates Dr. Neal Mahutte, at the Montreal Fertility Centre. "There will be a deluge."

Each cycle of IVF will include a single harvest of eggs, insemination and covered IVF, will now compete with other eligible Ontarians for just a single round. Women are eligible for funded treatment only up to the day before their 43rd birthday; gay men of any age are eligible for IVF using an egg donor and surrogate.

Public funding is often justified because it comes as a package with increased regulation and quality assurance. Currently, there is no comprehensive legislation that regulates the sector in the province. But it is not clear whether the new program will establish any, despite being recommended by the expert panel. "This is the time to introduce this," says Gruben.

The panel also recommended better and more open data collection. Currently, data is collected and owned privately by IVF clinic medical directors and reporting is not mandatory. Alison Motluk, Toronto, Ont.

CMAJ 2016. DOI:10.1503/cmaj.109-5210 